\title{
High and fluctuating glucose levels increase the expression and secretion of interleukin-18 in mouse peritoneal macrophages
}

\author{
CHENCHEN ZHANG ${ }^{1,2^{*}}$, YAXIN BI ${ }^{1 *}$, GUOXI JIN $^{1}$, HUAIYONG GAN $^{3}$ and LEI YU ${ }^{1}$ \\ ${ }^{1}$ Department of Endocrinology, The First Affiliated Hospital of Bengbu Medical College, Bengbu, Anhui 233020; \\ ${ }^{2}$ Department of Endocrinology, Lu'an Traditional Chinese Medicine Hospital, Lu'an, Anhui 237008; \\ ${ }^{3}$ Department of Pathology, Bengbu Medical College, Bengbu, Anhui 233020, P.R. China
}

Received June 23, 2014; Accepted March 9, 2015

DOI: $10.3892 / \mathrm{mmr} .2015 .3753$

\begin{abstract}
Macrophages are involved in the progression of atherosclerosis by releasing pro-inflammatory cytokines. High levels of interleukin (IL)-18 are associated with an increased risk of developing diabetes and atherosclerosis. The present study aimed to investigate the association between IL-18, and high and fluctuating glucose levels in mouse peritoneal macrophages (MPMs), and to assess the involvement of the c-Jun N-terminal kinase (JNK) pathway in this association. The MPMs were exposed to 4, 8, 16, 24 and $32 \mathrm{mM}$ glucose for $6 \mathrm{~h}$, which was alternated to either $4 / 24 \mathrm{mM}$ glucose every $1.5 \mathrm{~h}$ for $6 \mathrm{~h}$, or to $32 \mathrm{mM}$ glucose for $3,6,12$ and $18 \mathrm{~h}$. The expression and secretion levels of IL-18 were detected using reverse transcription-quantitative polymerase chain reaction (RT-qPCR) and ELISA, respectively. High levels of glucose increased the expression and secretion levels of IL-18 in a dose-dependent manner ( $\mathrm{P}<0.05$, vs. $4 \mathrm{mM}$ glucose). This increase was more important in the cells exposed to fluctuating $4 / 24 \mathrm{mM}$ glucose every $1.5 \mathrm{~h}$ compared with the cells exposed to stable $24 \mathrm{mM}$ glucose (RT-qPCR, 0.78 \pm 0.05 , vs. 0.66 \pm 0.07 ; ELISA, $188.23 \pm 20.32$, vs. $143.16 \pm 13.07 \mathrm{pg} / \mathrm{ml} ; \mathrm{P}<0.05)$. The expression and secretion levels of IL-18 increased 8 and $12 \mathrm{~h}$ following exposure to high-glucose, and then decreased at $18 \mathrm{~h}(\mathrm{P}<0.05$, vs. $3 \mathrm{~h})$. Furthermore, SP600125, a JNK inhibitor, decreased the high-glucose-induced gene expression of IL-18 in a dose-dependent manner. Therefore, high and fluctuating levels of glucose may be associated with inflammation and diabetic atherosclerosis by regulating the expression levels of IL-18. The present study identified the JNK signaling pathway as one of the mechanisms underlying this association.
\end{abstract}

Correspondence to: Mr. Guoxi Jin, Department of Endocrinology, The First Affiliated Hospital of Bengbu Medical College, 287 Changhuai Road, Bengbu, Anhui 233020, P.R. China

E-mail: 279740551@qq.com

*Contributed equally

Key words: macrophage, interleukin 18, glucose, atherosclerosis, diabetes, inflammation
Targeting IL-18 may be a novel therapeutic approach against diabetes-associated atherosclerosis.

\section{Introduction}

Diabetes is a prevalent metabolic disorder, characterized by increased levels of blood glucose (GLU). Diabetic macrovascular disease, characterized by atherosclerosis, is a chronic complication observed in patients with diabetes and is the predominant contributor to morbidity and mortality rates in patients with diabetes (1). Type 2 diabetes is considered equivalent to a previous history of myocardial infarction (MI) in terms of the MI risk stratification. The risk of MI in patients with Type 2 diabetes is $20 \%$, compared with $19 \%$ in patients with a history of $\mathrm{MI}$, and coronary mortality rates are $15 \%$, compared with $16 \%$ in patients with a history of MI (2). Dyslipidemia and inflammation are important in atherosclerosis (3). Activated macrophages are involved in the progression of atherosclerosis, the formation of foam cells and in inflammatory reactions (4). Interleukin (IL)-18 and IL-6 are important in the chronic inflammatory response and are primarily produced by macrophages (5-8).

Binding of IL-18 to the IL-18 receptor (IL-18R) directly activates nuclear factor- $\kappa \mathrm{B}(\mathrm{NF}-\kappa \mathrm{B})$ and stimulates $\mathrm{T}$-helper (Th) 2 responses (IL-4 and IL-13) (9) and IL-5 and IL-10 (10). IL-18 also increases cell adhesion molecules (11), nitric oxide synthesis (12), chemokine production (11) and induction of the Fas ligand (13). IL-18 is associated with obesity (14), metabolic syndrome (15), polycystic ovary syndrome (16), insulin resistance (17) and the risk of developing diabetes (18). IL-18 may also be a specific biomarker for atherosclerosis-prone patients with metabolic syndrome (19). Il-18 is a clear predictor of coronary mortality in patients with MI (20) and IL-18 polymorphisms are associated with an increased risk of MI (21). Therefore, the expression of IL-18 may be indicative of diabetic atherosclerosis.

In diabetic patients, GLU levels are rarely sustained throughout an entire day and they fluctuate according to the fasting state during the night and the successive postprandial states during the day (22). Previous studies have demonstrated that fluctuation in GLU levels during postprandial periods trigger higher levels of oxidative stress, inflammation and a higher risk of heart failure, compared with chronically 
sustained hyperglycemia (23-25). Blunting these fluctuations improves the levels of oxidative stress and inflammation in patients with type 2 diabetes (26). A previous in vivo study demonstrated that postprandial GLU fluctuations increased monocyte adhesion in the rat aorta (27). Fat cells exposed to GLU fluctuations exhibited increased expression levels of IL-18 (28), therefore, GLU fluctuations may be involved in diabetic macrovascular complications.

The c-Jun $\mathrm{NH}_{2}$-terminal kinase (JNK) pathway is involved in oxidative stress and inflammation (29). JNK is primarily activated by cytokines and by various cellular stresses (29). The activation of JNK induces the phosphorylation of c-Jun, which, in turn, activates the activator protein-1 (AP-1) transcription factor, which controls the expression levels of a number of genes, including IL-18 $(30,31)$.

However, the associations between high and fluctuating GLU levels, JNK, macrophages and IL-18 remain to be elucidated. Consequently, the present study assessed the expression and secretion levels of IL-18 in mouse peritoneal macrophages (MPMs) exposed to high and fluctuating concentrations of GLU, and examined the involvement of the JNK pathway in this association using SP600125, a JNK inhibitor.

\section{Materials and methods}

Animals. Male Kunming mice ( $\mathrm{n}=6$; pathogen-free, 5-7 weeks old) weighing 20-25 g were obtained from the Experimental Animal Center of Bengbu Medical College (Anhui, China). The mice were housed under standard conditions (12 h/day light; $25^{\circ} \mathrm{C}$ ) with free access to a basal diet (cat. no. GB14924.3-2001; Vital River Limited Company, Beijing, China) and water. All procedures and animal experiments were approved by the Animal Care and Use Committee of Bengbu Medical College (Anhui, China; Approval no. 2011001).

Isolation and purification of MPMs. The MPMs were isolated, as described previously (8). Briefly, each mouse was injected with $2 \mathrm{ml} \mathrm{6 \%}$ starch broth (Sigma-Aldrich, St. Louis, MO, USA). The abdomen was repeatedly rubbed for 2 min following injection, and the injection was repeated the following morning ( $12 \mathrm{~h}$ later). Following eye phlebotomy to reduce the quantity of blood in the animal and to prevent blood contamination of the peritoneal cavity, the mice were administered katamine (10 mg/ml, $0.2 \mathrm{ml} /$ mouse; Jiangsu Hengrui Medicine Co., Ltd., Jiangsu, China) and sacrificed by cervical dislocation. The skin was wiped with $75 \%$ alcohol (Sinopharm Chemical Reagent Co., Ltd., Shanghai, China), and pre-cooled phosphate-buffered saline $(10 \mathrm{ml})$ was injected intraperitoneally. Peritoneal fluid $(9 \mathrm{ml})$ was collected from each mouse and centrifuged for $15 \mathrm{~min}$ at $250 \mathrm{xg}$ at $4^{\circ} \mathrm{C}$ to obtain the MPMs. The MPM suspension was inoculated into cell culture plates $\left(2.5 \times 10^{6}\right.$ cells $\left./ \mathrm{ml}\right)$ and cultured in RPMI-1640 medium (KeGen BioTech, Nanjing, China), containing 10\% fetal calf serum (KeyGen Biotech. Co., Ltd., Nanjing, China) for $3 \mathrm{~h}$ in a $5 \% \mathrm{CO}_{2}$ incubator at $37^{\circ} \mathrm{C}$. The non-adherent cells were subsequent removed by gently washing the plates four times with D-Hanks' balanced salt solution. The MPMs were identified by hematoxylin and eosin (H\&E) staining and lysozyme immunocytochemical staining (Kecai Bio-engineering Limited Company. Shanghai, China).
Briefly, the tissue sections were deparaffinized twice in xylene for $10 \mathrm{~min}$ each and then re-hydrated twice in absolute alcohol (5 min each time), in 95\% alcohol for $2 \mathrm{~min}$ and in $70 \%$ alcohol for $2 \mathrm{~min}$. The tissue sections were washed briefly in distilled water and stained in Harris hematoxylin solution for $8 \mathrm{~min}$ and were subsequently washed in running tap water for $5 \mathrm{~min}$ and incubated in $1 \%$ acid alcohol for $30 \mathrm{sec}$. The tissue sections were washed in running tap water for $1 \mathrm{~min}$ and stained in $0.2 \%$ ammonia water or saturated lithium carbonate solution for $30 \mathrm{sec}$ to $1 \mathrm{~min}$. Sections were washed in running tap water for $5 \mathrm{~min}$ and rinsed ten times in $95 \%$ alcohol. The tissue sections were counterstained in eosin-phloxine solution for $30 \mathrm{sec}$ to $1 \mathrm{~min}$ and the sections were dehydrated using 95\% alcohol and twice in of absolute alcohol, 5 min each time. Finally the tissue sections were cleared twice in xylene for 5 min each time and mounted with xylene-based mounting medium. The tissue sections were visualized using a microscope (Olympus BX41; Olympus, Tokyo, Japan).

Study design. The purified MPMs were cultured $\left(3 \times 10^{5}\right.$ cells $\left./ \mathrm{ml}\right)$ in RPMI-1640 medium, containing $4 \mathrm{mM}$ D-GLU (normal GLU; NG; KeyGen Biotech. Co., Ltd.), supplemented with $10 \%$ heat-inactivated fetal calf serum (KeyGen Biotech. Co., Ltd.) and penicillin (100 U/ml; KeyGen Biotech. Co., Ltd.) for $12 \mathrm{~h}$. The MPMs were treated with specific concentrations of GLU for $6 \mathrm{~h}$ to form six groups with varying GLU conditions: 1) Continuous $4 \mathrm{mM}$ GLU; 2) continuous $8 \mathrm{mM}$ GLU; 3) continuous $16 \mathrm{mM}$ GLU; 4) continuous $24 \mathrm{mM}$ GLU; 5) continuous $32 \mathrm{mM}$ GLU and 6) alternating $4 \mathrm{mM}$ GLU and $24 \mathrm{mM}$ GLU every $1.5 \mathrm{~h}$. The MPMs were also treated with $32 \mathrm{mM}$ GLU for 3, 6, 12 or $18 \mathrm{~h}$. In additional experiments, MPMs $\left(3 \times 10^{5}\right.$ cells $\left./ \mathrm{ml}\right)$ were pretreated for $30 \mathrm{~min}$ with either SP600125 JNK inhibitor (Selleckchem, Houston, TX, USA), dissolved in 100\% dimethyl sulfoxide (DMSO; Sigma-Aldrich), and then diluted in culture medium or an equal volume of $0.1 \%$ DMSO as a negative control. The MPMs were then incubated at $37^{\circ} \mathrm{C}$ in $32 \mathrm{mM}$ D-GLU (high GLU; $\mathrm{HG}$ ) for $12 \mathrm{~h}$.

Reverse transcription-quantitative polymerase chain reaction (RT-qPCR). Total RNA was extracted from the MPMs using TRIzol reagent (Tiangen Biotech Co., Ltd., Beijing, China), and the RNA purity was determined by measuring the absorbance at 260 and $280 \mathrm{~nm}$ (A260/280) using a U-3900 UV spectrophotometer (Hitachi, Tokyo, Japan). The RNA was reverse transcribed into cDNA using SuperScript II reverse transcriptase and oligo $\mathrm{d}(\mathrm{T})$ (Tiangen Biotech Co., Ltd.), according to the manufacturer's instructions. Primer sequences for IL-18 and $\beta$-actin were designed, based on published sequence data from the NCBI database (http://www. ncbi.nlm.nih.gov/). The primers were synthesized by Sangon Biotech (Shanghai, China). All reactions were performed in triplicate. The primer sequences were as follows: IL-18, forward 5'-ATGGAGACCTGGAATCAGACA-3' and reverse 5'-CTTCACAGAGAGGGTCACAGC-3' (218 bp) and $\beta$-actin, forward 5'-TGGAATCCTGTGGCATCCATGAAAC-3' and reverse 5'-TAAAACGCAGCTCAGTAACAGTCCG-3' (349 bp). RT-qPCR was performed using the following cycle conditions: $94^{\circ} \mathrm{C}$ for $10 \mathrm{~min}, 94^{\circ} \mathrm{C}$ for $30 \mathrm{sec}$ and 30 cycles of $60^{\circ} \mathrm{C}$ for $30 \mathrm{sec}$ and $72^{\circ} \mathrm{C}$ for $45 \mathrm{sec}$, using a Stepone Plus 


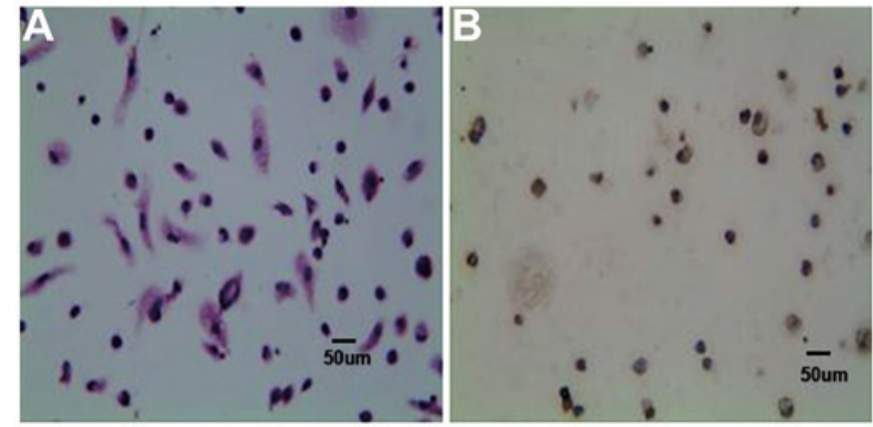

Figure 1. Identification of mouse peritoneal macrophages, which were confirmed based on (A) hematoxylin and eosin staining (magnification, $\mathrm{x} 400$ ) and (B) lysozyme positivity by immunocytochemistry (magnification, x400), as captured by Olympus BX41 light microscopy.

system (Applied Biosystems, Foster City, CA, USA). The PCR products were separated by $1.4 \%$ agarose (BioWest Agarose, Madrid, Spain) gel electrophoresis and scanned for grayscale intensity using the FluorChem HD2 Multifunction digital gel imaging analysis system (ProteinSimple, Santa Clara, CA, USA) with AlphaView 1.2.1.0 Image Analysis software (ProteinSimple). The relative mRNA expression levels of IL-18 were normalized against $\beta$-actin.

IL-18 release determination using ELISA. The cells were seeded into 96-well plates with $200 \mu \mathrm{l}$ medium, containing $2 \times 10^{6} \mathrm{MPMs} / \mathrm{ml}$. Following the different GLU treatments, each collected culture medium was centrifuged at $250 \mathrm{x} \mathrm{g}$ at $4^{\circ} \mathrm{C}$ to separate the floating cells, and the supernatant was stored at $-80^{\circ} \mathrm{C}$. The levels of IL-18 were measured using a specific mouse IL-18 immunoassay kit (Wuhan Boster Bio-engineering Limited Company, Wuhan, China).

Statistical analysis. The data are expressed as the mean \pm standard deviation. The data were analyzed using SPSS 16.0 software (SPSS Inc., Chicago, IL, USA). Normally distributed variables were analyzed by analysis of variance, with Fisher's least significant difference test for post hoc analysis. $\mathrm{P}<0.05$ was considered to indicate a statistically significant difference.

\section{Results}

Identification of MPMs. The presence of MPMs was confirmed based on H\&E staining (Fig. 1A) and on lysozyme positivity using immunocytochemistry (Fig. 1B). The purity of the MPM culture was $>95 \%$. The morphology of the inactive MPMs was round or oval, however, when the MPMs were stimulated with $6 \%$ starch broth, they exhibited extended pseudopodia, with numerous brown particles or vacuoles in the cytoplasm and darkly staining nuclei (Fig. 1A).

High and fluctuating levels of GLU increase the expression and secretion levels of IL-18 in a dose-dependent manner in MPMs. High GLU levels increased the mRNA expression and secretion levels of IL-18 in a dose-dependent manner (Fig. 2). The gene expression levels of IL-18 were increased 8-, 5.1-, 3.2- and 1.9-fold following treatment with 32, 24, 16 and $8 \mathrm{mM}$ D-GLU, respectively, compared with $4 \mathrm{mM}$ D-GLU

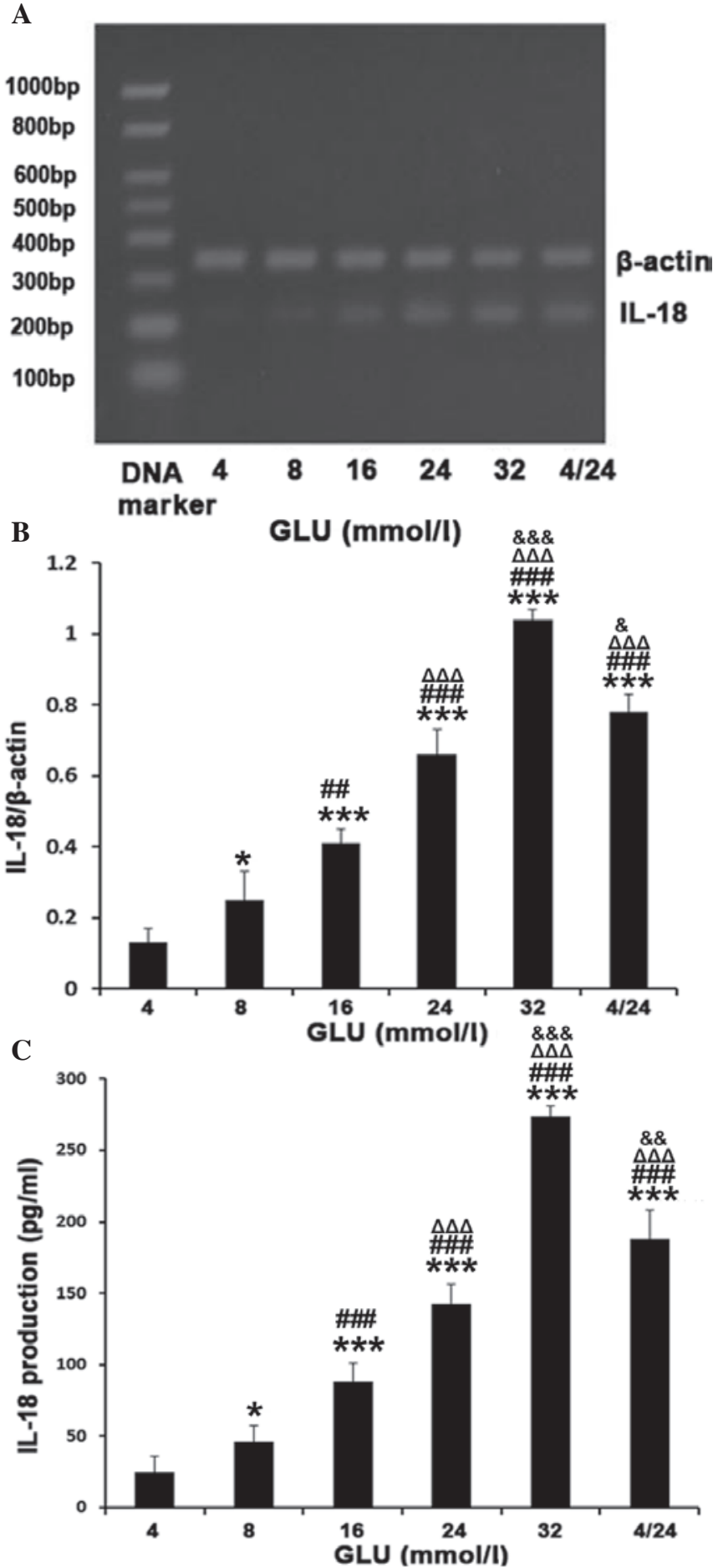

Figure 2. GLU increased the expression levels and secretion of IL-18 in a dose-dependent manner in MPMs. The MPMs were treated with different concentrations of GLU, including continuous treatment with either 4, 8 , 16,24 or $32 \mathrm{mM}$ GLU, or 4 and $24 \mathrm{mM}$ GLU fluctuating every $1.5 \mathrm{~h}$ for $6 \mathrm{~h}$. (A and B) The mRNA expression levels were determined by reverse transcription-quantitative polymerase chain reaction. $\beta$-actin was used as an internal reference. (C) The secretion of IL-18 was determined by ELISA. The results are expressed as the mean \pm standard deviation from three independent experiments $\left({ }^{*} \mathrm{P}<0.05\right.$ and ${ }^{* * *} \mathrm{P}<0.001$, vs. $4 \mathrm{mM} \mathrm{GLU;}{ }^{\# \#} \mathrm{P}<0.01$ and

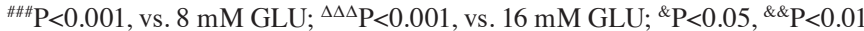
and ${ }^{\& \& \& P}<0.001$, vs. 24 mM GLU). GLU, glucose; MPMs, mouse peritoneal macrophages; IL, interleukin.

(all $\mathrm{P}<0.01$; Fig. 2A and B). The protein levels of IL-18 in the medium demonstrated similar results to those of the mRNA 

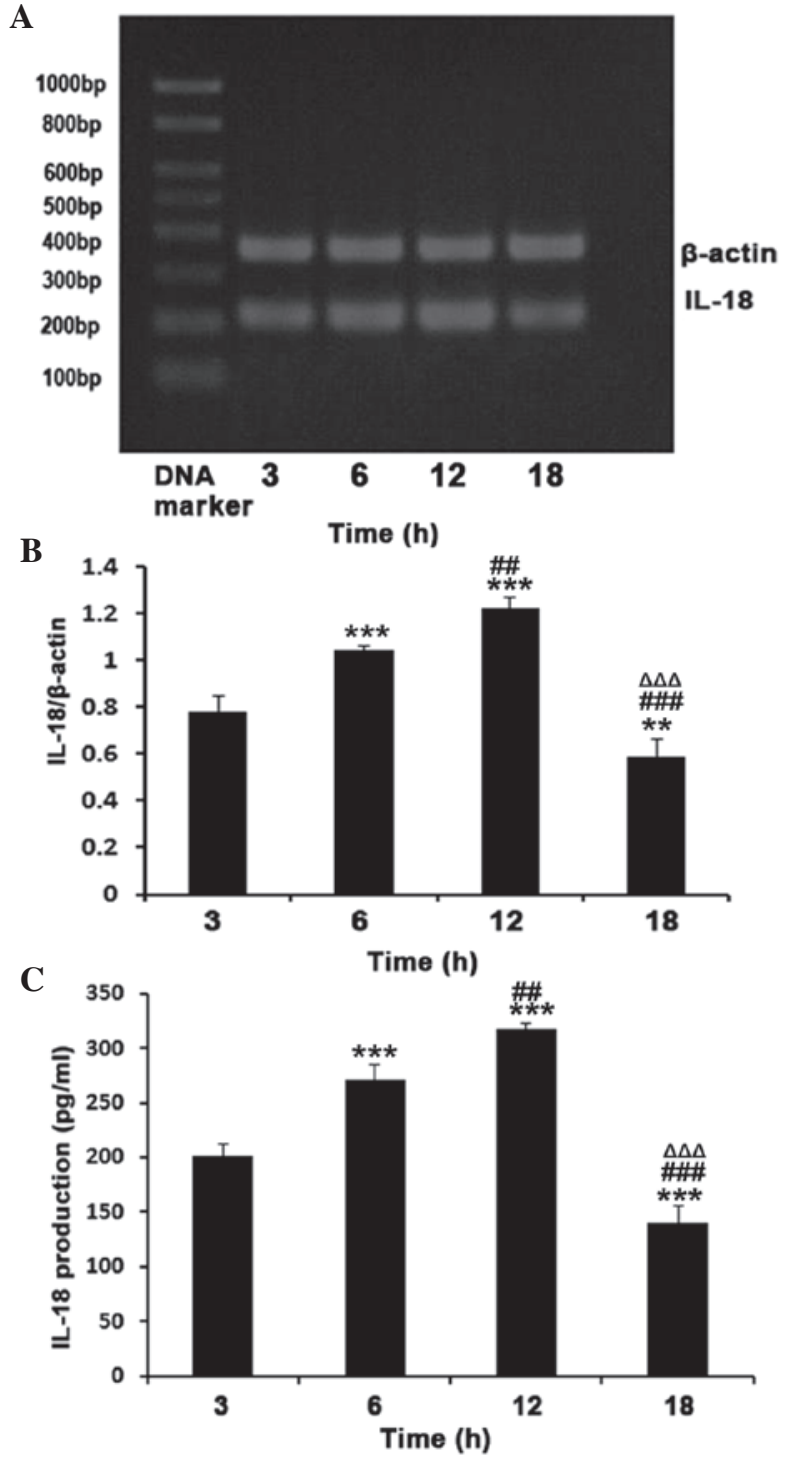

Figure 3. GLU increases the expression and secretion levels of IL-18 in a time-dependent manner in MPMs. The MPMs were treated with $32 \mathrm{mM} \mathrm{GLU}$ for 3, 6, 12 and $18 \mathrm{~h}$. (A and B) mRNA expression levels were determined by reverse transcription-quantitative polymerase chain reaction, using $\beta$-actin as an internal reference. (C) Secretion of IL-18 was determined by ELISA. The results are expressed as the mean \pm standard deviation from three independent experiments $\left({ }^{* *} \mathrm{P}<0.01\right.$ and ${ }^{* * *} \mathrm{P}<0.01$, vs. $3 \mathrm{~h}$; ${ }^{\# \#} \mathrm{P}<0.01$ and ${ }^{\# \# \#} \mathrm{P}<0.001$, vs. $6 \mathrm{~h}$ and ${ }^{\Delta \Delta \Delta} \mathrm{P}<0.05$, vs. $12 \mathrm{~h}$ ). GLU, glucose; IL, interleukin; MPMs, mouse peritoneal macrophages.

(Fig. 2C). Compared with stable $24 \mathrm{mM}$ GLU, this increase was more notable when the GLU levels fluctuated between 4 and $24 \mathrm{mM}$ every $1.5 \mathrm{~h}$ (RT-qPCR, 0.78 \pm 0.05 , vs. 0.66 \pm 0.07 ; ELISA, $188.23 \pm 20.32$, vs. $143.16 \pm 13.07 \mathrm{pg} / \mathrm{ml} ; \mathrm{P}<0.05)$ as shown in Fig. 2.

Effects of the duration of high GLU treatment on the expression and secretion of IL-18 in MPMs. A significant increase in the mRNA expression and secretion levels of IL-18 were observed following $3 \mathrm{~h}$ treatment with $32 \mathrm{mM}$ D-GLU (HG), following which levels of IL-18 continued to increase and reached a maximum at $12 \mathrm{~h}$. At $18 \mathrm{~h}$, the levels decreased compared with those observed at $3 \mathrm{~h}(\mathrm{P}<0.01$; Fig. 3). Following HG treatment for $6 \mathrm{~h}$ and $12 \mathrm{~h}$, the mRNA expression levels of IL-18 increased 1.3- and 1.6-fold, respectively compared with $3 \mathrm{~h}$ treatment
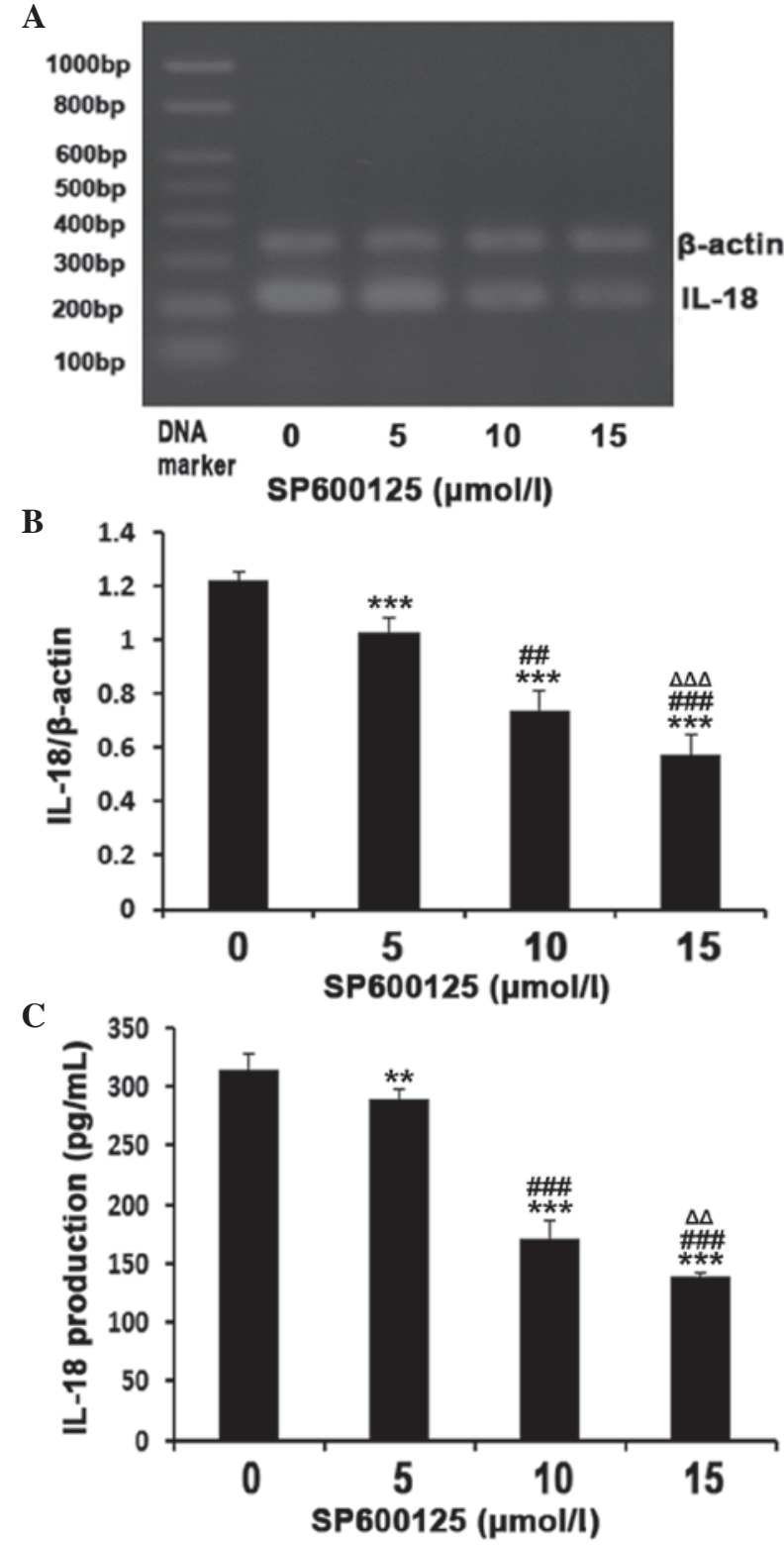

Figure 4. Inhibitory effect of the JNK inhibitor, SP600125, on the $32 \mathrm{mM}$ GLU-induced mRNA expression and secretion of IL-18 in MPMs. The MPMs were pre-treated with different concentrations of SP600125 for $30 \mathrm{~min}$, and the cells were subsequently incubated with $32 \mathrm{mM}$ GLU in the presence of SP600125 at the indicated doses. (A and B) mRNA expression levels were determined by reverse transcription-quantitative polymerase chain reaction, using $\beta$-actin as an internal reference. (C) Secretion of IL-18 was determined by ELISA. The results are expressed as the mean \pm standard deviation from three independent experiments $\left({ }^{* * *} \mathrm{P}<0.05\right.$ and ${ }^{* * *} \mathrm{P}<0.01$, vs. $0 \mu \mathrm{M}$ SP600125; ${ }^{\# \#} \mathrm{P}<0.05$ and ${ }^{\# \# \#} \mathrm{P}<0.001$, vs. $5 \mu \mathrm{M}$ SP 600125 and ${ }^{\Delta \Delta} \mathrm{P}<0.05$ and ${ }^{\Delta \Delta \Delta} \mathrm{P}<0.001$, vs. $10 \mu \mathrm{M}$ SP600125). GLU, glucose; IL, interleukin; MPMs, mouse peritoneal macrophages; JNK, c-Jun N-terminal kinase.

$(\mathrm{P}<0.01)$. There was a significant decrease at $18 \mathrm{~h}$, with a $24.4 \%$ reduction compared with that at $3 \mathrm{~h}(\mathrm{P}<0.01$; Fig. $3 \mathrm{~A}$ and $\mathrm{B})$. The secretion of IL-18 followed the same trend as the mRNA expression levels (Fig. 3C).

Role of JNK in HG-induced expression and secretion of IL-18 in MPMs. Treatment with the JNK inhibitor, SP600125, significantly inhibited the HG-induced expression and secretion levels of IL-18 in a dose-dependent manner (Fig. 4). The reductions in 
the mRNA expression levels of IL-18 were 15.6, 39.3 and 53.3\% when pretreated with 5, 10 and $15 \mu \mathrm{M}$ SP600125, respectively, compared with the MPMs treated with $\mathrm{HG}$ alone as a negative control ( $\mathrm{P}<0.01$; Fig. 4A and B). The protein levels of IL-18 in the medium followed the same trend (Fig. 4C).

\section{Discussion}

Previous studies have demonstrated that the expression of IL-18 is increased in metabolic syndrome (15), diabetes, obesity (14), and polycystic ovary syndrome (16). It has also been revealed that IL-18 is associated with the development of vascular lesions and diabetic atherosclerosis (32). Notably, diabetes (18) and cardiovascular diseases (33) have been associated with higher expression levels of IL-18 at baseline, therefore, IL-18 may be involved in the development of diabetes-associated atherosclerosis.

The present study investigated the effects of different concentrations of GLU on the secretion and mRNA expression levels of the proinflammatory cytokine, IL-18, in MPMs. The results demonstrated that $\mathrm{HG}$ induced a significant increase in the secretion and mRNA expression levels of IL-18, and these effects occurred in a dose-dependent manner. In addition, GLU fluctuations significantly promoted the expression and secretion of IL-18 compared with stable GLU concentrations. It was further demonstrated that the expression and secretion levels of IL-18 were increased at 8 and $12 \mathrm{~h}$ following exposure to $\mathrm{HG}$, and decreased at $18 \mathrm{~h}$. These results suggested that the effects of GLU on IL-18 were mediated by the JNK pathway, since SP600125, a JNK inhibitor, decreased the production of IL-18 production in a dose-dependent manner. However, comprehensive pathway analysis is required to elucidate the underlying mechanisms.

It has been well established that GLU increases the expression levels of a number of cytokines, including IL-6, IL-12 and IL-18, 2.0-fold at a $10 \mathrm{mM}$ concentration of GLU, and that this effect is dose-dependent (8). Compared with previous studies, the present study used lower concentration increments of GLU, providing refined details on the GLU-mediated expression of IL-18. The highest concentration used, $32 \mathrm{mM}$, induced the highest expression levels of IL-18, suggesting that using higher concentrations of GLU may induce higher expression levels of IL-18.

By contrast to a previous study by Wen et al (8), where the authors used constant GLU levels only, the present study included a fluctuating GLU group, in which GLU levels alternated between 4 and $24 \mathrm{mM}$ every $1.5 \mathrm{~h}$, simulating postprandial cycles. In this group, the mRNA expression levels of IL-18 were higher compared with the stable $14 \mathrm{mM}$ group. A previous study demonstrated a significant increase in the expression levels of IL-18 in fat cells exposed to GLU fluctuations (28). These results and those of the present study suggested that the stimulatory function of GLU fluctuation on IL-18 is more significant than stable GLU concentrations. These results concur with results previously observed in patients (23-25), suggesting that patients may benefit from strict glycemic control to maintain GLU levels as stable as possible.

The present study demonstrated that the HG-induced mRNA expression of IL-18 occurred as early as $3 \mathrm{~h}$, peaked at $12 \mathrm{~h}$ and decreased at $18 \mathrm{~h}$. However, a previous study revealed that the peak occurred at $3 \mathrm{~h}$, which differed from the results of the present study (8). This discrepancy may be due to a number of factors. Firstly, the controls were not identical, as the present study examined time course of IL-18 at only one GLU concentration, whereas the previous study measured the expression of IL-18 in different concentration groups at a single time-point. Secondly, a number of factors may affect the expression of IL-18 other that GLU concentration, and these factors may not have been controlled in the previous study. Previous animal and clinical investigations have demonstrated the existence of a peak in the expression of IL-18 under different stresses $(8,28,34,35)$, and the results of the present study are consistent with these studies, even if the peak did not occur at the same time-point.

IL-18 is well known to be modulated by inflammation (31,36-38). In addition, clinical trials have demonstrated that plasma levels of IL-18 correlate with insulin resistance (17). JNK belongs to the mitogen-activated protein kinase (MAPK) family and contributes to inflammatory responses, cell proliferation, apoptosis and tissue morphogenesis $(29,39)$. A previous study reported that JNK inhibitors significantly reduce the LPS-stimulated expression of IL-18 in murine keratinocytes (40). Another study revealed that HG stimulated the activation of protein kinase C, p38, MAPK-p38, JNK and nuclear transcription factor-kB kinase, and that inhibitors of these pathways all reduce the mRNA expression levels of IL-12 in MPMs (8). To examine whether the JNK pathway is involved in the effects of HG on IL-18, the present study used SP600125, a highly selective inhibitor of JNK1, JNK2 and JNK3, which was observed to inhibit the HG-induced expression of IL-18 in a dose-dependent manner. These results suggested that the JNK pathway was involved in the HG-induced mRNA expression of IL-18.

The present study had several limitations. Firstly, whether the expression levels of IL- 8 in MPMs peak at the same time-point in different concentrations of GLU was not investigated. Secondly, further investigations are required to elucidate the association between the HG-induced expression of IL-18 and JNK inhibitors and the duration of treatment. Furthermore, the underlying mechanisms remain to be elucidated. As a proinflammatory cytokine, the overexpression of IL-18 is not an isolated event in the human body, and further investigation is required to evaluate the association between IL-18 and other proinflammatory factors, including IL-1, IL-6, IL-12, IL-18, tumor necrosis factor- $\alpha$ and interferon- $\gamma$, all of which are significantly increased under $\mathrm{HG}$ (8).

In conclusion, HG increased the expression and secretion of IL-18 in MPMs in a dose-dependent manner. GLU fluctuations had an impact on the expression and secretion of IL-18, and the JNK signaling pathway may be a mechanism involved in the effects of HG. These results suggested that HG-induced expression and secretion of IL-18 in macrophages may be involved in the development of diabetes and long-term vascular complications.

\section{Acknowledgements}

This study was supported by the Natural Science Foundation of the Anhui Higher Education Institutions, China (no. KJ2014A156) and Health Department issues of Anhui province (no. 09A014). 


\section{References}

1. Kannel WB and McGee DL: Diabetes and cardiovascular disease. The Framingham study. JAMA 241: 2035-2038, 1979.

2. Haffner SM, Lehto S, Rönnemaa T, et al: Mortality from coronary heart disease in subjects with type 2 diabetes and in nondiabetic subjects with and without prior myocardial infarction. N Engl J Med 339: 229-234, 1998.

3. Hansson GK: Inflammation, atherosclerosis and coronary artery disease. N Engl J Med 352: 1685-1695, 2005.

4. Shalhoub J, Falck-Hansen MA, Davies AH and Monaco C: Innate immunity and monocyte-macrophage activation in atherosclerosis. J Inflamm (Lond) 8: 9, 2011

5. Formigli L, Manneschi LI, Nediani C, et al: Are macrophages involved in early myocardial reperfusion injury? Ann Thorac Surg 71: 1596-1602, 2001.

6. Herskowitz A, Choi S, Ansari AA and Wesselingh S: Cytokine mRNA expression in postischemic/reperfused myocardium. Am J Pathol 146: 419-428, 1995.

7. Lotz M: Interleukin-6. Cancer Invest 11: 732-742, 1993.

8. Wen Y, Gu J, Li SL, et al: Elevated glucose and diabetes promote interleukin-12 cytokine gene expression in mouse macrophages. Endocrinology 147: 2518-2525, 2006.

9. Nakanishi K, Yoshimoto T, Tsutsui $\mathrm{H}$ and Okamura $\mathrm{H}$ : Interleukin-18 is a unique cytokine that stimulates both Th1 and Th2 responses depending on its cytokine milieu. Cytokine Growth Factor Rev 12: 53-72, 2001.

10. Hoshino T, Kawase Y, Okamoto M, et al: Cutting edge: IL-18-transgenic mice: in vivo evidence of a broad role for IL-18 in modulating immune function. J Immunol 166: 7014-7018, 2001.

11. Pope RM and Tschopp J: The role of interleukin- 1 and the inflammasome in gout: implications for therapy. Arthritis Rheum 56: $3183-3188,2007$

12. Hoshino K, Tsutsui H, Kawai T, et al: Cutting edge: generation of IL-18 receptor-deficient mice: evidence for IL-1 receptor-related protein as an essential IL-18 binding receptor. J Immunol 162: 5041-5044, 1999.

13. Tsutsui H, Nakanishi K, Matsui K, et al: IFN-gamma-inducing factor up-regulates Fas ligand-mediated cytotoxic activity of murine natural killer cell clones. J Immunol 157: 3967-3973, 1996.

14. Esposito K, Pontillo A, Ciotola M, et al: Weight loss reduces interleukin-18 levels in obese women. J Clin Endocrinol Metab 87: 3864-3866, 2002.

15. Hung J, McQuillan BM, Chapman CM, et al: Elevated interleukin-18 levels are associated with the metabolic syndrome independent of obesity and insulin resistance. Arterioscler Thromb Vasc Biol 25: 1268-1273, 2005.

16. Escobar-Morreale HF, Botella-Carretero JI, Villuendas G, et al: Serum interleukin-18 concentrations are increased in the polycystic ovary syndrome: relationship to insulin resistance and to obesity. J Clin Endocrinol Metab 89: 806-811, 2004.

17. Fischer CP, Perstrup LB, Berntsen A, et al: Elevated plasma interleukin-18 is a marker of insulin-resistance in type 2 diabetic and non-diabetic humans. Clin Immunol 117: 152-160, 2005.

18. Thorand B, Kolb H, Baumert J, et al: Elevated levels of interleukin-18 predict the development of type 2 diabetes: results from the MONICA/KORA Augsburg Study, 1984-2002. Diabetes 54: 2932-2938, 2005

19. Yamaoka-Tojo M, Tojo T, Wakaume K, et al: Circulating interleukin-18: A specific biomarker for atherosclerosis-prone patients with metabolic syndrome. Nutr Metab (Lond) 8: 3, 2011.

20. Blankenberg S, Tiret L, Bickel C, et al: Interleukin-18 is a strong predictor of cardiovascular death in stable and unstable angina. Circulation 106: 24-30, 2002

21. Tiret L, Godefroy T, Lubos E, et al: Genetic analysis of the interleukin-18 system highlights the role of the interleukin-18 gene in cardiovascular disease. Circulation 112: 643-650, 2005.
22. Hansen AP and Johansen K. Diurnal patterns of blood glucose, serum free fatty acids, insulin, glucagon and growth hormone in normals and juvenile diabetics. Diabetologia 6: 27-33, 1970.

23. Monnier L, Mas E, Ginet C, et al: Activation of oxidative stress by acute glucose fluctuations compared with sustained chronic hyperglycemia in patients with type 2 diabetes. JAMA 295: 1681-1687, 2006

24. Chang CM,Hsieh CJ,Huang JC and Huang IC: Acute and chronic fluctuations in blood glucose levels can increase oxidative stress in type 2 diabetes mellitus. Acta Diabetol 49: S171-S177, 2012.

25. Node K and Inoue T: Postprandial hyperglycemia as an etiological factor in vascular failure. Cardiovasc Diabetol 8: 23, 2009.

26. Rizzo MR, Barbieri M, Marfella R and Paolisso G: Reduction of oxidative stress and inflammation by blunting daily acute glucose fluctuations in patients with type 2 diabetes: role of dipeptidyl peptidase-IV inhibition. Diabetes Care 35: 2076-2082, 2012.

27. Azuma K, Kawamori R, Toyofuku Y, et al: Repetitive fluctuations in blood glucose enhance monocyte adhesion to the endothelium of rat thoracic aorta. Arterioscler Thromb Vasc Biol 26: 2275-2280, 2006.

28. Sun J, Xu Y, Dai Z and Sun Y: Intermittent high glucose stimulate MCP-1, IL-18 and PAI-1, but inhibit adiponectin expression and secretion in adipocytes dependent of ROS. Cell Biochem Biophys 55: 173-180, 2009.

29. Gill R, Tsung A and Billiar T: Linking oxidative stress to inflammation: Toll-like receptors. Free Radic Biol Med 48: 1121-1132, 2010.

30. Davis RJ: Signal transduction by the JNK group of MAP kinases. Cell 103: 239-252, 2000.

31. Kim YM, Im JY, Han SH, et al: IFN-gamma up-regulates IL-18 gene expression via IFN consensus sequence-binding protein and activator protein-1 elements in macrophages. J Immunol 165: 3198-3205, 2000

32. Mallat Z, Corbaz A, Scoazec A, et al: Interleukin-18/interleukin-18 binding protein signaling modulates atherosclerotic lesion development and stability. Circ Res 89: E41-E45, 2001.

33. Everett BM, Bansal S, Rifai N, et al: Interleukin-18 and the risk of future cardiovascular disease among initially healthy women. Atherosclerosis 202: 282-288, 2009.

34. Arndt PG, Fantuzzi G and Abraham E: Expression of interleukin-18 in the lung after endotoxemia or hemorrhage-induced acute lung injury. Am J Respir Cell Mol Biol 22: 708-713, 2000.

35. Esposito K, Nappo F, Marfella R, et al: Inflammatory cytokine concentrations are acutely increased by hyperglycemia in humans: role of oxidative stress. Circulation 106: 2067-2072, 2002.

36. Kim YM, Kang HS, Paik SG, et al: Roles of IFN consensus sequence binding protein and PU.1 in regulating IL-18 gene expression. J Immunol 163: 2000-2007, 1999.

37. Alsaleh G, Suffert G, Semaan N, et al: Bruton's tyrosine kinase is involved in miR-346-related regulation of IL-18 release by lipopolysaccharide-activated rheumatoid fibroblast-like synoviocytes. J Immunol 182: 5088-5097, 2009.

38. Cordoba-Rodriguez R, Fang H, Lankford CS and Frucht DM: Anthrax lethal toxin rapidly activates caspase-1/ICE and induces extracellular release of interleukin (IL)-1beta and IL-18. J Biol Chem 279: 20563-20566, 2004.

39. Zhu J, Krishnegowda G and Gowda DC: Induction of proinflammatory responses in macrophages by the glycosylphosphatidylinositols of Plasmodium falciparum: the requirement of extracellular signal-regulated kinase, p38, c-Jun N-terminal kinase and NF-kappaB pathways for the expression of proinflammatory cytokines and nitric oxide. J Biol Chem 280: 8617-8627, 2005.

40. Yun W and Li C: JNK pathway is required for TNCB-induced IL-18 expression in murine keratinocytes. Toxicol In Vitro 24: 1064-1069, 2010. 